\title{
$495-20280$
}

\section{AIAA 92-4711 \\ An Efficient Constraint to Account for Mistuning Effects in the Optimal Design of Engine Rotors}

Durbha V. Murthy

The University of Toledo

Toledo, Ohio

Christophe Pierre and Gisli Ottarsson The University of Michigan Ann Arbor, Michigan

Fourth

AIAA/USAF/NASA/OAI

Symposium on Multidisplinary Analysis and Optimization $\$$

September 21-23, 1992 / Cleveland, $\mathrm{OH}$ 


\title{
AN EFFICIENT CONSTRAINT TO ACCOUNT FOR MISTUNING EFFECTS IN THE OPTIMAL DESIGN OF ENGINE ROTORS
}

\author{
Durbha V. Murthy' \\ The University of Toledo, Toledo, ahio \\ Christophe Pierre ${ }^{\mathrm{t}}$ \\ The University of Michigan, Ann Arbor, Michigan \\ Gísli Óttarsson" \\ The University of Michigan, Ann Arbor, Michigan
}

\begin{abstract}
$\underline{\text { Abstract }}$
Blade-to-blade differences in structural properties, unavoidable in practice due to manufacturing tolerances, can have significant influence on the vibratory response of engine rotor blades. Accounting for these differences, also known as mistuning, in design and optimization procedures is generally not possible. This note presents an easily calculated constraint that can be used in design and optimization procedures to control the sensitivity of final designs to mistuning.
\end{abstract}

\section{Introduction}

Vibration of blades is an important concern in the design of gas turbine engines. The demands of high thrustto-weight ratios, compact size, high fuel efficiency and increased life and reliability in modem propulsion systems are increasingly leading to performance limits imposed by vibration phenomena, such as flutter and forced response. Past and current experience with space propulsion systems in the Space Shuttle Main Engine and the proposed National Launch System (NLS), as well as with the aeropropulsion systems such as the propfans, ultra-high-bypass ducted-fan engines and conventional turbofans demonstrates the importance of blade vibration behavior in the development of turbomachinery.
Nearly all design procedures for engine blades, and indeed most analyses of dynamic behavior, are based on the routine assumption that all blades on a rotor are identical. This assumption of perfect cyclic symmetry in engine rotors, while customary, is only approximately true in practice. Small differences in structural, material and aerodynamic properties among the blades, commonly referred to as mistuning, are unavoidable in practice because they arise from manufacturing tolerances and in-service degradation. The implicit assumption in most of the design procedures is that the effect of practical levels of blade-to-blade differences is not significant on the vibratory response of the blades.

That this assumption could be profoundly wrong has been demonstrated by several studies which investigated the influence of mistuning on blade assembly dynamic behavior. $^{1-8}$ It was concluded that while mistuning is generally beneficial for flutter, the adverse effects of mistuning on forced response can generally be significant and sometimes even drastic. It has been demonstrated that even small levels of mistuning could result in large increases (of the order of several hundred percent) in the maximum forced response amplitudes. When such is the case, the assumption of perfect cyclic symmetry, often called the tuned rotor assumption, gives highly misleading results. This sensitivity to mistuning can be particularly dangerous when automated optimization procedures are employed in design, because an optimal design that is extremely sensitive to mistuning may result, invalidating the entire optimization process in practice.

An obvious and direct approach to account for the adverse effects of mistuning is to model a mistuned rotor

" Resident Research Associate, NASA Lewis Research Center, Cleveland, Ohio, Senior Member AIAA.

† Associate Professor, Dept. of Mechanical Engineering and Applied Mechanics, Member AIAA.

** Graduate Research Assistant, Dept. of Mechanical Engineering and Applied Mechanics 
and place constraints on the amplitudes of individual blades to excitations of interest. However, this approach presents a number of difficulties. First, tremendous analytical complexities arise because it is no longer sufficient to model the dynamic behavior of a single blade but modeling of the entire blade assembly having relatively high modal density is required. Second, the size of the analysis problem increases several fold (by a factor equal to the number of blades), leading to the multiplication of analysis and sensitivity analysis computational times and the number of constraints by the same factor. Third and most important, the mistuning is random by nature and the actual mistuning pattern is not available until the design and manufacture of the rotor are complete. Mistuning also differs from rotor to rotor. Moreover, the mistuning that results from in-service degradation is practically impossible to model deterministically. Thus, even a full scale mistuned analysis cannot be usefully performed in a deterministic manner.

In this note, we suggest a way out of this impasse. Our approach is based on the realization that the tuned rotor assumption, in spite of its limitations, is extremely valuable in analyses and, optimization procedures, because of the associated analytical simplifications and computational advantages. Given this situation, we note that it would be valuable to an optimization procedure if a constraint that restricts the sensitivity of a rotor design to mistuning were available, without the need for an extensive analysis of the entire mistuned rotor.

The specific objective of this note is to present a strategy to develop such a constraint for an engine rotor and illustrate the approach by applying it to a model popularly used to analyze bladed-disk vibrations. The proposed constraint is dependent on the properties of only the tuned system, so a mistuned system analysis is not needed. We overcome the principal difficulty in modeling mistuning by employing a statistical model, which does not require knowledge of the actual mistuning pattern. This is practical because the statistics of expected mistuning may be more readily obtained or estimated than the actual mistuning pattern. The complex and time-consuming task of a mistuned system analysis is avoided by employing perturbation theory in deriving the constraint. As a result, the proposed constraint is very inexpensive to compute. This constraint is also easy to differentiate, sensitivity analysis of the constraint imposes no additional burden, provided a sensitivity analysis of the tuned assembly is already available. Thus, it is suitable for the computationintensive applications of optimization of engine rotors. The principal limitation of the proposed approach is that aerodynamic coupling is not accounted for. However, this limitation may be removed by further work.
The outline of the note is as follows. The adverse effects of mistuning are first related to the phenomenon of vibration localization. A localization factor that quantifies vibration localization is then defined. This localization factor is employed in the development of a constraint that is useful in accounting for mistuning without a full mistuned analysis. An explicit expression for the localization factor is given for a generic bladed-disk model.

\section{Mistuning and Vibration Localization}

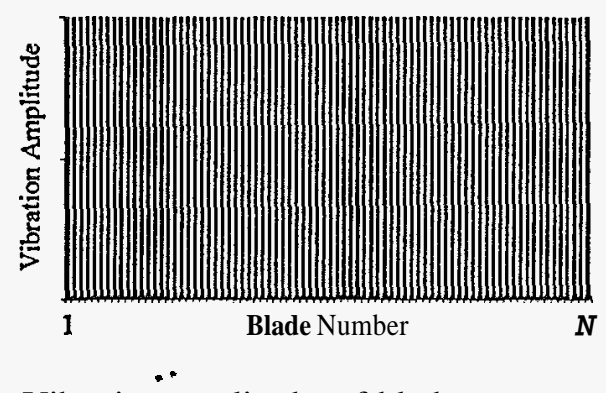

Figure 1 - Vibration amplitudes of blades on a tuned rotor.

A well-known consequence of the tuned rotor assumption is that all the blades on a tuned rotor vibrate with identical amplitudes when subjected to a dynamic excitation which is also perfectly cyclic symmetric. Thus the energy of excitation is shared equally among all the blades, which obviously minimizes fatigue. Figure 1 schematically illustrates the vibration amplitudes of blades on a tuned rotor, a pattern that is generally the goal of the designer.

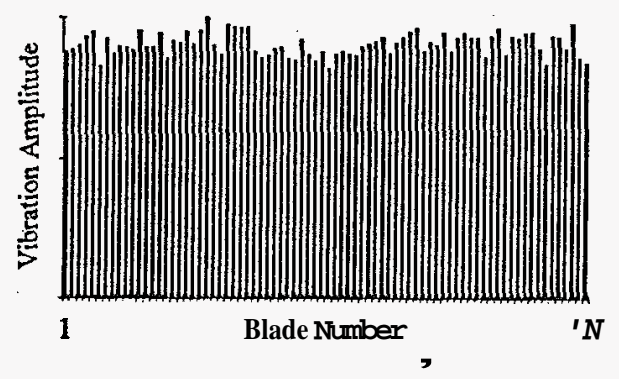

Figure 2 - Vibration amplitudes of blades on a rotor with very low mistuning.

Mistuning introduces blade-to-blade differences in blade properties and destroys the cyclic symmetry of the rotor. Hence mistuning also results in blade-to-blade differences in the amplitudes of vibration. If the level of mistuning is sufficiently small, then all or most blades still participate in the vibration, even though the amplitudes are not identical any longer, as shown in the schematic of Figure 2. 


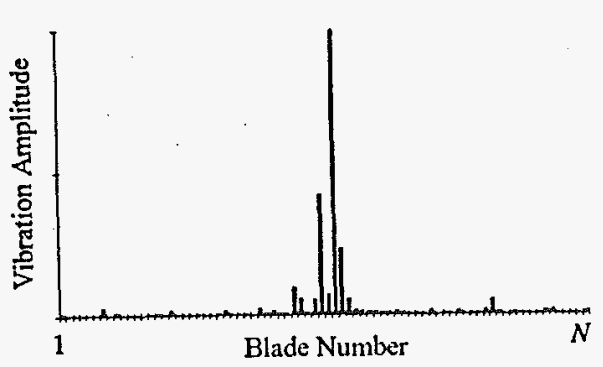

Figure 3 - Localized vibration pattern of a mistuned rotor.

However, if the level of mistuning is sufficiently high, there could be a drastic change in the distribution of the vibration amplitudes. When this happens, vibration is constrained to very few blades of the rotor, in a phenomenon is known as mode localization, as depicted (schematically) in Figure 3. Thus, all the energy of the excitation is absorbed by a small number of blades, resulting in very high amplitudes which can lead to failure or shortened life. Note that the localization phenomenon can never be predicted by conventional tuned system analyses. Thus, a design optimized for stability and response using tuned system assumptions can actually turn out to have far larger amplitudes of vibration than anticipated.

The strength of vibration localization at a given frequency, and consequently the possibility of large vibratory response, is determined by the sensitivity to blade mistuning at that frequency. In general, this sensitivity is a function of the tuned (i.e., average or nominal) characteristics of the system.

In the next section, we outline a strategy to quantify vibration localization so it may be constrained in design procedures.

\section{The Mistuning Constraint}

Although information about localization in the blade assembly's natural modes would be invaluable, a statistical description of the modes of vibration is elusive due to the intricate shifting of the associated natural frequencies as the mistuning strength is varied. Hence, we choose to examine instead the propagation of free waves in the mistuned assembly. This gives us full control of the frequency at which we wish to study the localization.

The constraint we propose is based .on the localizationfactor derived in Reference 9. The development is briefly repeated here and the reader is referred to Reference 9 for full details.

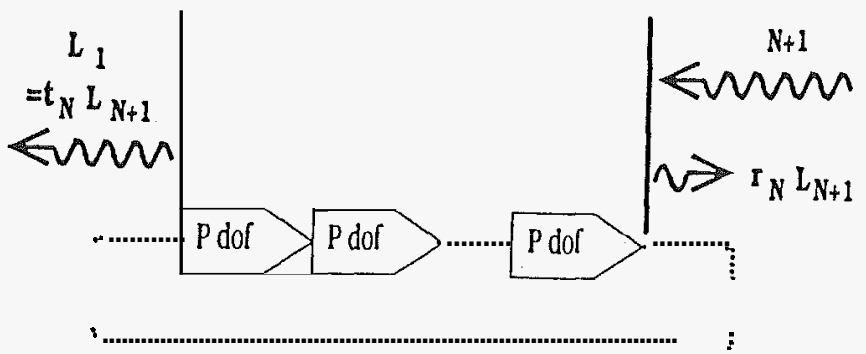

Figure 4 - A general N-bay nearly cyclic assembly with $\boldsymbol{P}$ degrees of freedom per bay, one of which couples adjacent bays.

The bladed disk is modeled as an $N$-bay nearly cyclic assembly with $\boldsymbol{P}$ degrees of freedom per bay, one of which couples adjacent'bays (a mono-coupled system) as in Figure 4. It is well known that in perfectly 'periodic mono-coupled structures there exists only one pair of wave modes at each frequency, one traveling clockwise and one traveling counterclockwise." Furthermore, there are bands of frequencies, known as passbands, in which these waves travel with no attenuation. Vibrations of the structure may be expressed in terms of these wave modes through a two dimensional wave transfer matrix which relates wave mode amplitudes at adjacent blades. The wave transfer matrix for a series of blades is the product of the wave transfer matrix for each blade and for a tuned assembly is diagonal since the clockwise traveling wave is independent of the counterclockwise traveling wave.

In a mistuned blade assembly, however, the wave transfer matrix is not diagonal. A possibility of localization (standing wave) exists even in the frequency passbands, because the clockwise and counterclockwise wave modes can no longer travel unattenuated through the structure. Instead a wave is partially reflected as it traverses each boundary between the dissimilar blades in the assembly. The left incident wave $L_{N_{+1}}$ to the structure (see Figure 4 ) is partially reflected (by a factor equal to the reflection coefficient $r_{N}$ ) and the emergent wave $L_{1}$ is equal to $t_{N} L_{N+l}$, where $t_{N}$ is the transmission coefficient for the structure at that frequency. Making now the assumption that, on average, the ratio of emergent to incident wave decreases exponentially with an increasing number of bays, we define a frequency-dependent localization factor $\gamma$ as the ratio of the amplitudes of the incident wave $L_{N+1}$ and the emergent wave $\boldsymbol{L}$, or, for an assembly of $N$ mistuned blades,

$\left(\frac{L_{N+1}}{L_{1}}\right)=e^{\gamma N}$

or, 


$$
\gamma N=\left\langle\ln \left|\frac{1}{t_{N}}\right|\right.
$$

where Odenotes the average or expected value. Averaging is used to eliminate explicit dependence on mistuning pattern. Note that the localization factor defines the average exponential decay rate per bay and thus is a descriptor of the strength of localization.

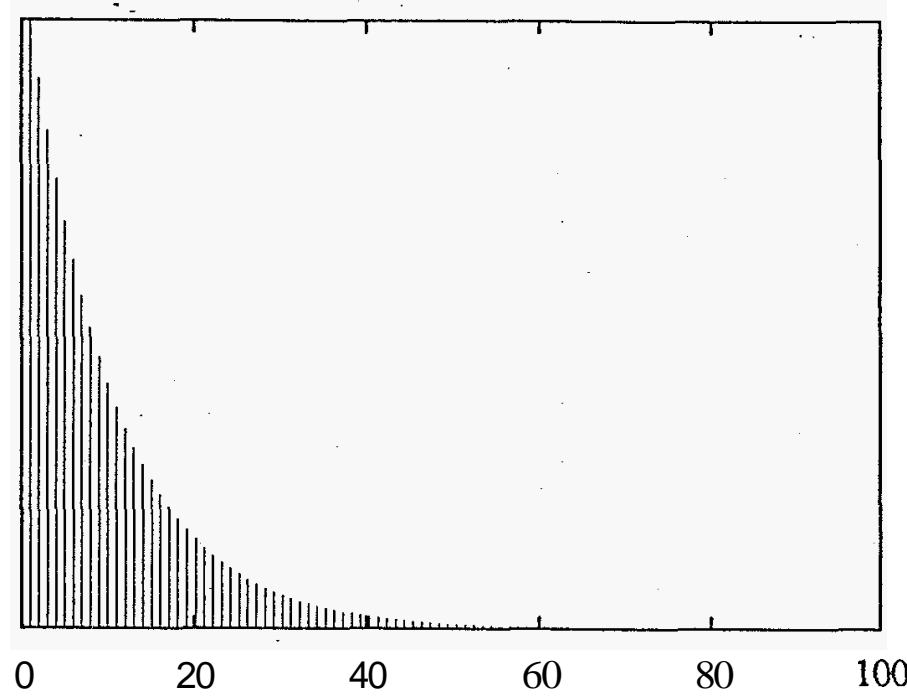

Figure 5 - An idealized 100 blade assembly with localization factor $\gamma=0.1$.

Let us illustrate the use of the localization factor through some examples. Recall that $\gamma$ is defined as the average spatial exponential decay rate of a wave propagating through the structure at some frequency. The attenuation is governed by $e^{-\gamma N}$. Hence, the larger the value of $\gamma$ the sharper the attenuation. For $\gamma=0.1$, the ratio of the amplitudes of adjacent bays is $e^{-0.1} \approx 0.90$ such that on average the energy transmitted from one blade to the next is $82 \%$ and $\mathbf{5 6 \%}$ is transmitted to the 3 rd blade. 'The amplitude would be attenuated by $90 \%$ by blade 24. Figure 5 depicts a wave subject to localization with $\gamma=0.1$. For $\gamma=1$ the average energy transmitted to the next blade is $13.5 \%$ and less than $0.25 \%$ of the energy reaches the 3rd blade! Of course, for the tuned, undamped system $\gamma=0$ and $100 \%$ of the energy is transmitted.

From Figure 5, we see that the significance of the value of $\gamma$ depends on the total number of blades in the assembly. To be completely meaningful the localization factor must be used in conjunction with the total number of blades, $\boldsymbol{N}$. Obviously the localization in Figure 5 would appear much less significant in a 10-bay assembly than it would in a 100-bay assembly. In the former case $\gamma N=1$ but $\gamma N=10$ in the latter.

This suggests constraining $\gamma N$ to a suitable value to limit the effects of localization in a mistuned bladeassembly. For instance, one might require a wave propagating through the system to retain at least $80 \%$ of its amplitude, on average, thus:

$$
e^{-\gamma^{N}} \geq 0.8
$$

or

$$
\gamma N+\ln 0.8 \leq 0
$$

Note that the evaluation of the constraint in eq. (4) requires the calculation of the expected value or the averaging of the localization factor $\gamma$. An obvious but costly solution to generating numerical values for the localization factor is the Monte Carlo simulation method. This is a "brute force" method in which a series of realizations of mistuned assemblies are generated using a random number generator. The exponential decay rate of an incident wave' is calculated for each realization. The localization factor is the average of the result for the multiple realizations. The method is expensive due to the high number of realizations usually required for adequate accuracy. Furthermore, its cost is compounded by the fact that the localization factor must be obtained at all frequencies of interest. An alternative to Monte Carlo simulations is an analyticalapproximation of the localization factor via perturbation techniques. We illustrate this approach in the next section by applying it to a popularly used bladed-disk dynamic model.

\section{Illustration}

The concepts above are illustratedby applying them to a common blade assembly model. Dye and Henry' were among the first to propose this blade assembly model, which was later studied extensively by several authors. We base much of the following development on the work by Óttarsson and Pierre'.

The blade assembly model is shown in Figure 6. Note that there are two degrees of freedom per bay: one for the single-mode motion of the blade and one for motion of the disk at the blade root, denoted $q_{i}^{b}$ and $q_{i}{ }^{d}$ respectively. Corresponding to $q_{i}^{b}$ are the blade modal mass $m_{b}$ and modal stiffness $k_{b}^{i}$. The mass $m_{d}$ simulates the effective 


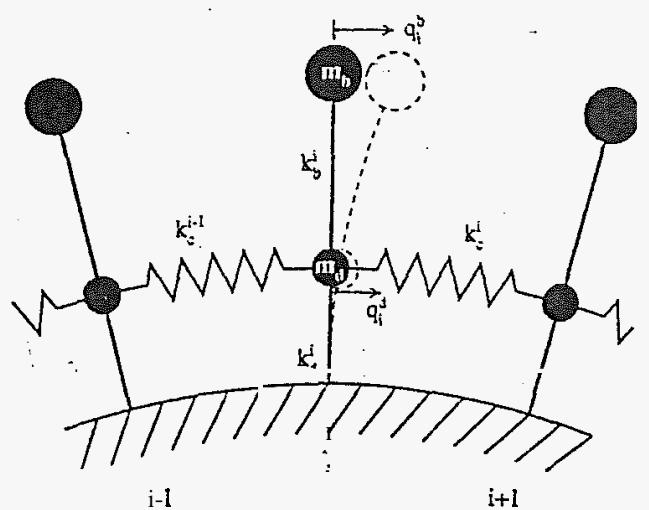

i-i

$\mathrm{i}+\mathrm{i}$

Figure 6 - The ith blade in an' $\mathrm{N}$-blade assembly with one blade coordinate and one disk coordinate per bay is shown with its two coupled adjacent blades.

mass of the blade root and the corresponding section of the disk. The stiffness $k_{d}^{i}$ represents the basic stiffness of the rotor disk and $k_{c}{ }^{i}$ provides the disk coupling between adjacent blades. We make the common assumption that mistuning affects only the blade stiffness $k_{b}^{i}$.

The natural frequencies of the tuned system represented by this model are given by

$$
\bar{\omega}_{n}^{2}=\frac{\bar{K}+\bar{m}+1 \pm \sqrt{(\bar{K}+\bar{m}+1)^{2}-4 m R}}{2 \bar{m}}
$$

where $n=.1, . .$, Nand

$$
\begin{aligned}
& \bar{K}=2 \bar{k}_{c}\left(1-\cos \sigma_{n}\right)+\bar{k}_{d} \\
& \sigma_{n}=\frac{2 \pi(n-1)}{N} \\
& m=m_{d} / m_{b}
\end{aligned}
$$

and the non-dimensional quantities $\bar{k}_{d}$ and $\bar{k}_{c}$ are the average values of $k_{d}{ }^{\prime}, k_{c}{ }^{\prime}$ respectively non-dimensionalized by $m_{b}$. The minus sign in eq. (5) is used for the first band of frequencies and the plus sign for the second. 'There are two bands of frequenciesbecause our model has two degrees of freedom per periodic bay.

It is customary in practice to plot the natural frequency distribution against the number of nodal diameters in the corresponding mode of vibration. This is done in

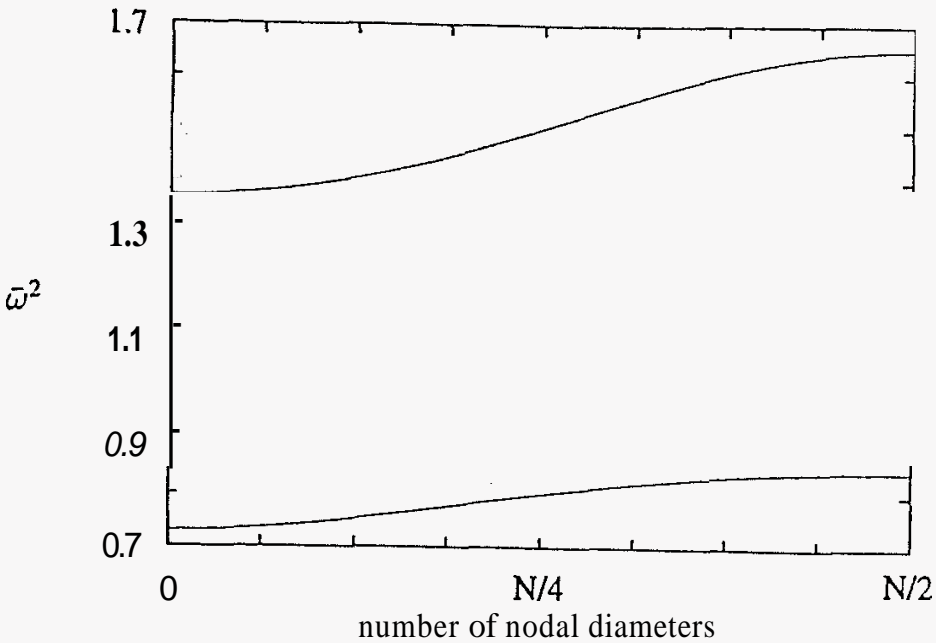

Figure 7 - Natural frequencies as a function of nodal diameter for the bladed-disk model in Figure 6.

Figure 7 for $\boldsymbol{i}_{d}=10, \tilde{k}_{c}=1$ and $\bar{m}=10$ for our model. Note that even quite complicated bladed disk models can be reduced to the model used in the present study simply by fitting the actual natural frequency distribution to the distribution such as shown in Figure 7 and identifying the values of $\dot{k}_{d} \bar{k}_{c}$ and $\bar{m}$ for the best fit. Reference 11 provides an example of such a procedure.

Recall that $\gamma$ is a function of natural frequency. In other words, the localization phenomenon could manifest in different strengths in differentrotor modes. However, in an optimization procedure, it would be too cumbersometo place constraints on the localization factors on each rotor mode. Any convenient or "interesting" mode can be chosen for this purpose. For convenience, we place the constraint on the mode corresponding to. $\sigma=\pi / 2$. Note that the frequency corresponding to this mode is the median natural frequency (i.e., there are as many natural frequencies above it as there are below) and in that sense this mode may be considered "typical". The localization factor of the system can be then written as

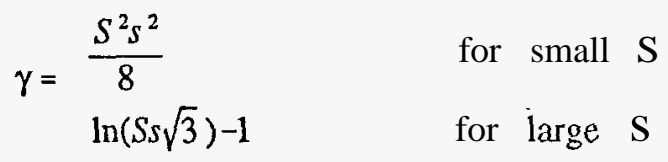

where $s$ is the maximum standard deviation of the mistuning in blade stiffness and $S$ is defined by 
$S=$

$\frac{1}{4 \bar{k}_{c}}\left[2 \bar{k}_{c}+\bar{k}_{d}-\bar{m}-1 \mp \sqrt{\left(w \bar{k}_{c}+\bar{k}_{d}+\bar{m}+1\right)^{2}-4 \bar{m}\left(\bar{k}_{d}+2 \bar{k}_{c}\right)}\right]^{2}$

Note that the eqs. (7) express $\gamma$ as a discontinuous function of the bladed disk modal parameters. This could be a severe limitation in optimization applications. However, this limitation could be easily overcome by redefining y as

$$
\begin{array}{lr}
\gamma=\frac{\dot{S}^{2} s^{2}}{8} & \text { for } S<30 \\
y=\frac{1}{\dot{h}}[(S-80)+2(S-30)]-\frac{1}{h^{3}}\left[(S-80)^{3}+(S-30)^{3}\right] \\
\gamma=\ln (S s \sqrt{3})-1 & \text { for } 30 \leq S \leq 80 \\
& \text { for } S>80
\end{array}
$$

using a spline interpolation between the two segments of eqs. (7). The numbers 30 and 80 in eq. (9) were chosen to match well with the Monte Carlo results.

The constraint on the influence of mistuning can now be similar to eq. ((4)).

\section{Concluding Remarks}

In this note, we outline a strategy to constrain the adverse effects of random blade mistuning in the design of engine rotors. The proposed constraint can be evaluated from tuned system properties and the expected level of mistuning in the rotor. The constraint is based on limiting the localization of a wave incident to the structure. We illustrate the evaluation of the localization factor using a common bladed-disk assembly model.

A limitation of the niodel chosen for illustration in this note is that only structural coupling (i.e., no aerodynamic coupling is considered. However, the development of the constraint would be simply analogous for a model that accounts for structural as well as aerodynamic coupling. For example, a similar constraint can be easily developed based on the sensitivity measure for the model that accounts for only aerodynamic coupling*.

\section{$\underline{\text { References }}$}

1. Dye, R. C. and Henry, T. A., "Vibration Amplitudes of Compressor Blades Resulting from Scatter in Blade Natura Frequencies", Journal of Engineering for Power, 1969, pp. 182-188.
2. Ewins, D. J., "Vibration Characteristics of Bladed Disc Assemblies", Journal of Engineering Science, vol. 15, No. 3, 1973, pp. 165-186.

3. El-Bayoumy, L. E. and Srinivasan, A. V., "Influence of Mistuning on Rotor-Blade Vibrations", AIAA Journal, Vol. 13, No. 4, 1975, pp. 460-464.

4. Kielb, R. E. and Kaza, K. R. V., "Effects of Structural Coupling on Mistuned Cascade Flutter and Response", Journal of Engineering for Gas Turbines and Power, Vol. 106, No. 1, 1984, pp. 17-24.

5. Ewins, D. J. and Han, Z. S., "Resonant Vibration Levels of a Mistuned Bladed Disk", Journal of Vibration, Acoustics, Stress and Reliability in Design, Vol. 106, 1984, pp. 211 217.

6. Valero, N. A. and Bendiksen, O. O., "Vibration Characteristics of Mistuned Shrouded Blade Assemblies",Joumal of Engineering for Gas Turbines and Power, Vol. 108, 1986, pp. 293-299.

7. Wei, S. T. and Pierre, C., :Localization Phenomena in Mistuned Assemblies with Cyclic Symmetry, Part I: Free Vibrations", Journal of Vibration, Acoustics, Stress and Reliability in Design, Vol. 110, 1988, pp. 293-299.

8. Pierre, C. and Murthy, D. V., "Aeroelastic Modal Characteristics of Mistuned Blade Assemblies: Mode Localization and Loss of Eigenstructure", presented at the AIAA/ASME/ASCE/AHS/ASC 32nd Structures, Structural Dynamics and Materials Conference, Baltimore, Maryland, April 8-10, 1991.

9. Óttarsson, G and Pierre, $C_{\text {A }}$ " A Transfer Matrix Assemblies", Preprint to be published in the proceedings of the AIAA/ASME/ASCE/AHS/ASC 34th Structures, Structural Dynamics and Materials Conference, La Jolla, California, April 19-21, 1993.

10. Mead, D. J., "Wave Pronagation and Natural Modestił,
Periodic Systems, I: Mono-coupled Systems", pp. 1-18.

11. Griffin, J. H. and Hoosac, T. M., "Model Development and Statistical Investigation of Turbine Blade Mistuning", Journal of Vibration, Stress and Reliability in Design, Vol. 106, 1984, pp. 204-210. 